\title{
TV/Series
}

Hors séries 1 | 2016

Lost: (re)garder l'île

\section{« We have to go back! » : (re)garder l'île}

\section{Claire Cornillon and Sarah Hatchuel}

\section{(2) OpenEdition}

\section{Journals}

\section{Electronic version}

URL: http://journals.openedition.org/tvseries/1737

DOI: 10.4000/tvseries.1737

ISSN: 2266-0909

\section{Publisher}

GRIC - Groupe de recherche Identités et Cultures

\section{Electronic reference}

Claire Cornillon and Sarah Hatchuel, « « We have to go back! » : (re)garder l'île », TV/Series [Online], Hors séries 1 | 2016, Online since 03 October 2016, connection on 23 September 2020. URL : http:// journals.openedition.org/tvseries/1737 ; DOI : https://doi.org/10.4000/tvseries.1737

This text was automatically generated on 23 September 2020

\section{(c) (i) () $\Theta$}

TV/Series est mis à disposition selon les termes de la licence Creative Commons Attribution - Pas d'Utilisation Commerciale - Pas de Modification 4.0 International. 


\title{
«We have to go back! »: (re)garder l'île
}

\author{
Claire Cornillon and Sarah Hatchuel
}

1 Un avion s'écrase sur une île du Pacifique. Perdus dans une zone inconnue du globe, quelques rescapés découvrent que l'île n'est pas inhabitée et que d'étranges phénomènes s'y produisent. Ensemble, ils vont affronter les Autres, conjurer leur passé tourmenté et décrypter les mystères de l'île. Dans ses expérimentations narratives, Lost ( $A B C, 2004-2010)$ nous fait partager la désorientation des personnages. La série brouille les repères temporels, met au défi notre compréhension de l'histoire et nous invite à un travail d'orientation à la fois dans le récit et dans nos vies. La série incite constamment à se retourner sur l'œuvre pour mieux l'appréhender, réconciliant postmodernisme et souci de sincérité, distance critique et émotion pure, visions du monde multiples et expérience universelle de vie et de mort. Histoire initiatique d'un éveil aux mystères de l'existence, la série multiplie les allers-retours géographiques et temporels, les rebondissements et les renversements de perspectives. Lost pense notre rapport à autrui, au temps, à la vérité, à la croyance et à la fiction. Série de coïncidences, de miracles et de retrouvailles, Lost a touché des millions de spectateurs et a tout simplement révolutionné la construction narrative télévisuelle.

2 On a pu penser que l'île de Lost en avait fini avec nous, et nous avec elle. Il n'en était rien. Depuis que Jack Shephard (Matthew Fox) a fermé l'œil pour la dernière fois le 23 mai 2010 sur $A B C$, les études sur Lost ne se sont pas éteintes, bien au contraire. Des ouvrages collectifs sont parus en anglais avant la fin de la série, tel Reading Lost: Perspectives on a Hit Television Show dirigé par Roberta Pearson (Londres, I. B. Tauris, 2009) ; d'autres juste après, comme Ultimate Lost and Philosophy: Think Together, Die Alone dirigé par Sharon Kaye (Hoboken, John Wylez \& Sons, 2011), Looking for Lost: Critical Essays on the Enigmatic Series dirigé par Randy Laist (Jefferson, McFarland, 2011) et Lost thought dirigé par Pearson Moore (Inukshuk press, 2012). En France, deux monographies sur la série sont parues - Les Mêmes yeux que Lost de Pacôme Thiellement (Paris, Éditions Léo Sheer, 2011) et Lost: Fiction vitale de Sarah Hatchuel (PUF, 2013), mais il manquait encore une exploration collective de l'œuvre, un regard 
kaléidoscopique posé sur la série afin de l'éclairer de multiples manières, à travers différentes approches et disciplines, tout comme la série tente de parvenir à un regard parfait posé sur ses personnages.

3 Nous avons souhaité que ce numéro soit évolutif pour qu'il puisse accueillir sans cesse de nouveaux articles, en français comme en anglais, et ainsi accompagner la recherche en marche sur Lost. Les vagues de publication successives viendront refléter, d'une certaine manière, l'œuvre sérielle, ses saisons et épisodes. Pour explorer, de manière jamais figée, une philosophie immanente à la série, mais aussi la tension qu'elle construit entre illusion et réalité ou encore le rapport au regard et à l'altérité qu'elle met en place, le numéro est construit autour de grandes sections qui viendront régulièrement s'enrichir de nouvelles contributions. Ces sections portent volontairement des titres issus de répliques qui ont marqué la série afin d'affirmer la volonté de ce numéro d'éclairer la série par une pensée conceptualisée, et non l'inverse. Il s'agit d'étudier la série comme œuvre en soi et non comme illustration d'une théorie.

4 La section « Not Penny's boat! » s'intéressera à l'expérience affective dans et de Lost. La section « Man of Science, Man of Faith » traitera des questions de doute et de foi, que se posent à la fois les personnages de fiction et les spectateur.rice.s de la série. La section «It's not easy being an Other » se concentrera sur la question de l'altérité. La section «See you in another life, brother! » étudiera les intertextes et influences de la série, à la fois dans son écriture et dans sa manière d'exister dans d'autres fictions. La section "Whatever happened happened» se focalisera sur les questions d'acceptation et de fatalisme à l'œuvre dans la série. Enfin, la section "Remember, let go, move on » explorera la fin, si controversée, d'une série qui compte six saisons et 121 épisodes.

D'autres sections pourront être ouvertes en fonction des contributions que nous recevrons.

6 De manière originale par rapport à ce qui se pratique généralement dans l'étude des séries télévisées, des séries d'articles pourront se concentrer sur un épisode en particulier, comme "Cabin Fever" (4.11) ou "LaFleur» (5.8), afin de l'analyser à travers des approches croisées et révéler à quel point un épisode peut aussi s'appréhender comme une œuvre et entretenir des relations complexes avec la série dans son ensemble.

7 Si, comme l'a montré Pacôme Thiellement, Lost joue le rôle, dans notre vie de spectateur.rices, de l'île dans la vie des personnages comme lieu de transcendance et d'orientation, alors ce numéro se veut un lieu d'analyse, d'exégèse et de méta-récit qui retourne la série à la vie et la redonne sans cesse à ses spectateur.rice.s afin qu'ils.elles prennent le relais d'Hurley et la (re)gardent au mieux. 


\section{AUTHORS}

\section{CLAIRE CORNILLON}

Claire Cornillon est docteur en littérature comparée. Elle est également titulaire d'un master en études cinématographiques. Elle est actuellement PRAG à l'Université Paul Valéry-Montpellier 3. Ses recherches les plus récentes portent sur la narration et la fiction dans les séries télévisées américaines, en particulier de network. Elle co-dirige la collection Sérial aux Presses Universitaires François Rabelais.

Claire Cornillon has a PhD in comparative literature and a master in film studies. She teaches at the Université Paul Valéry-Montpellier 3. Her latest research aims at studying fiction and narration in American TV series, especially network series. She is general co-editor of the «Sérial » collection published at the Presses Universitaires François Rabelais.

\section{SARAH HATCHUEL}

Sarah Hatchuel, Présidente de la Société Française Shakespeare, est Professeure en littérature et cinéma anglophones à l'université du Havre. Elle est l'auteure de livres sur Shakespeare au cinéma (Shakespeare and the Cleopatra/Caesar Intertext : Sequel, Conflation, Remake, Fairleigh Dickinson University Press, 2011; Shakespeare, from Stage to Screen, Cambridge University Press, 2004 ; A Companion to the Shakespearean Films of Kenneth Branagh, Blizzard Publishing, 2000) et sur les séries télévisées américaines (Rêves et séries américaines: la fabrique d'autres mondes, Rouge Profond, 2015 ; Lost, PUF, 2013). Elle a codirigé (avec Nathalie Vienne-Guerrin) huit volumes de la collection Shakespeare on Screen et codirige (avec Ariane Hudelet) la revue TV/Series.

Sarah Hatchuel is Professor of English Literature and Film at the University of Le Havre (France), President of the Société Française Shakespeare and head of the 'Groupe de recherché Identités et Cultures'. She has written extensively on adaptations of Shakespeare's plays (Shakespeare and the Cleopatra/Caesar Intertext: Sequel, Conflation, Remake, Fairleigh Dickinson University Press, 2011; Shakespeare, from Stage to Screen, Cambridge University Press, 2004; A Companion to the Shakespearean Films of Kenneth Branagh, Blizzard Publishing, 2000) and on TV series (Lost: Fiction vitale, PUF, 2013; Rêves et series américaines: la fabrique d'autres mondes, Rouge Profond, 2015). She is general editor of the CUP Shakespeare on Screen collection (with Nathalie Vienne-Guerrin) and of the online journal TV/Series (with Ariane Hudelet). 